\section{Ingeniería}

CONTENIDO

Artículos

Análisis numérico de un sistema de aire acondicionado empleando
mallas estructuradas y no estructurades A

Funciones de regresión para caudales extremos en la Vertiente Rojas, Nazareth; Aguilar, José Francisco; Solís, Hernán. Evaluación de daños por agresión ambiental en viviendas
de concreto reforzado. Solís, Rómel; Moreno, Eric; Jiménez, Felipe; Rosas, Victorino.

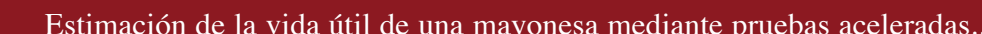
Estimación de la vida útil de una mayonesa
García, Claudia; Molina, Manuel.

Inestabilidad oscilatoria de tensión debido a los motores de inducción …….....................65-75 rde, Gustavo; Araya, Eddie.

Estabilidad y colapso de tensión en sistemas eléctricos Araya, Eddie.

Obtención de pulpa a la soda antraquinona de Tectona grandis creciendo en Costa Rica...........................

Protocolo evaluador para el manejo de desechos y la seguridad ocupacional
aplicado al laboratorio clínico del Hospital San Francisco de Asís de Grecia. Ruiz, Francisco.

9. Euler: su contex
Vargas, Celso.

10. Reserva de resistencia de edificios porticados de concreto armado disenaados conforme al ACI-318/ IBC 2006 ........ Análisis y comentarios

Herrera, Rodolfo.

breve semblanza.

Trabajos de Graduación 2007

Lista de proyectos de graduación de grado y posgrado

Normas

$\sum_{\text {EDTORAL }}$

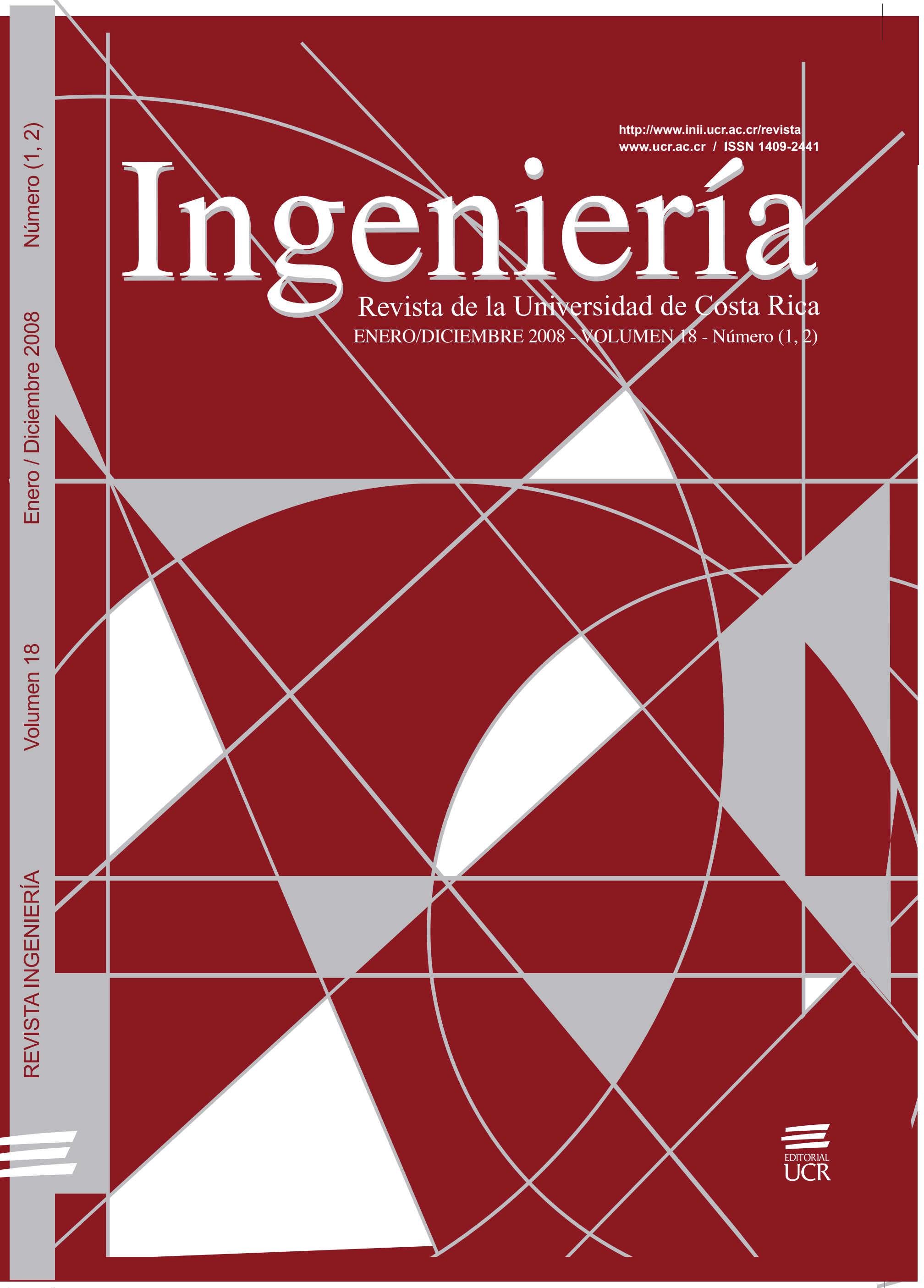




\title{
ESTABILIDAD Y COLAPSO DE TENSIÓN EN SISTEMAS ELÉCTRICOS CON TOPOLOGÍA LONGITUDINAL
}

Eddie A. Araya Padilla

\begin{abstract}
Resumen
Esta publicación analiza el fenómeno de la estabilidad y colapso de tensión en sistemas de potencia con topología longitudinal, similares al mexicano, italiano, taiwanés o centroamericano.

Se propone un modelo del sistema de potencia que permite incluir el efecto de dispositivos de compensación en derivación. Con base en este modelo, se determina el punto crítico de operación correspondiente a la condición de máxima cargabilidad del sistema. Dichos resultados son verificados a través del análisis de valores singulares de las ecuaciones de flujo de potencia.

La metodología desarrollada permite evaluar condiciones críticas de operación, antes de la manifestación de un colapso de tensión. Los resultados obtenidos, son útiles para determinar los estados de operación vulnerables de un sistema longitudinal, cuyos centros de consumo se encuentran lejos de las fuentes de energía eléctrica.
\end{abstract}

Palabras clave: estabilidad de tensión, cargabilidad, punto crítico de operación.

\begin{abstract}
This paper analyzed the phenomenon of the stability and collapse of voltage in longitudinal power systems, similar to the Mexican, Italian, Taiwan or Central American.

The model proposed allows including the effect of devices of compensation. With this model, the critical point determined the loadability of the system. The resulted are verified through the analysis of singular values of the equations of power flow.

The developed methodology allows evaluating critical conditions of operation, before the manifestation of a collapse of voltage in longitudinal power system
\end{abstract}

Keywords: voltage stability, loadability, critical point operation.

Recibido: 07 de noviembre del 2008 • Aprobado: 03 de diciembre del 2008

\section{INTRODUCCIÓN}

En los últimos años, la presencia de problemas asociados al control de tensión, ha aumentado en forma notable debido a una diversidad de factores, como por ejemplo, el crecimiento en el consumo de energía, en las políticas de diseño y operación actuales de los sistemas de potencia, las cuales dificultan la adquisición de servidumbres para nuevos enlaces de transmisión y propician una mayor utilización de la red eléctrica; o el efecto combinado de tales factores, reduciendo así, los márgenes de estabilidad de tensión.

El problema de estabilidad de tensión se relaciona, de manera amplia, con la capacidad del sistema para mantener niveles aceptables de tensión en todos los nodos del sistema, bajo condiciones normales de operación o después de una perturbación (Taylor, 1994). Sin embargo, un incremento considerable en la demanda 
o un cambio en las condiciones operativas y topológicas del sistema post-falla, podrían causar una caída progresiva e incontrolable de la tensión y el sistema entraría a una condición de inestabilidad o colapso (Stubbe et al., 1993).

El análisis de varios eventos en distintas redes eléctricas, muestra que el fenómeno de la inestabilidad de tensión, puede manifestarse en forma rápida que incluye aspectos dinámicos, o de manera más lenta en régimen permanente (Obadina \& Berg, 1990). En algunos casos, la pérdida de la estabilidad de tensión se presenta después de alguna contingencia en el sistema, principalmente la salida de operación de una línea de transmisión altamente cargada. En otros casos, sin embargo, la pérdida de cantidades significativas de potencia reactiva, por ejemplo asociados a la compensación estática de potencia reactiva, también puede conducir a la pérdida de estabilidad de tensión (Stubbe et al., 1993).

Este fenómeno puede tomar distintas formas y períodos de tiempo. Frecuentemente, los ángulos de fase y la frecuencia del sistema permanecen constantes y el fenómeno de inestabilidad de tensión se manifiesta fundamentalmente por la disminución incontrolable de la tensión del sistema, a menudo en un período largo de tiempo causando la operación del equipo de protección. En la etapa inicial, la magnitud de tensión en nodos críticos del sistema decae lentamente. La acción de los sistemas automáticos de control, puede en algunos casos mejorar de manera momentánea los perfiles de tensión, para posteriormente decrecer en forma incontrolable causando la operación del equipo de protección. Esta forma de inestabilidad se asocia con frecuencia a la acción o respuesta lenta del equipo de control secundario de tensión, esencialmente la acción de transformadores con cambio automático de carga, la conexión de bancos de capacitores y la naturaleza de la carga (Obadina \& Berg, 1990). Este fenómeno es frecuentemente descrito en la literatura como estabilidad de tensión en régimen permanente, para distinguirlo de otros fenómenos de inestabilidad (Obadina \& Berg, 1990).

El problema de la inestabilidad de tensión, también se ha manifestado en sistemas energéticos de países en vía de desarrollo. Estos sistemas de potencia caracterizados por la configuración radial de cargas, frecuentes desbalances de potencia activa y reactiva entre la generación y la demanda, un limitado número de líneas de transmisión, baja capacidad de cortocircuito del sistema y una densidad no uniforme de carga, son llamados sistemas de potencia longitudinales (SPL) (Aboytes \& Arroyo, 1986) o sistemas de potencia débiles (Abdel, 1987).

En el caso nacional, Costa Rica no esta inmune de este fenómeno, prueba de ello, el pasado 6 de marzo del 2001 a las 18:42 h, el país sufrió un colapso de tensión que afectó al $100 \%$ del Sistema Eléctrico Nacional. En esa ocasión, la energía no servida fue de 2500 MW-h (ICE, 2001).

En el pasado, el problema de la inestabilidad de tensión fue analizado en el extremo receptor de la línea, cuando la tensión nodal comienza a decaer por debajo de su valor nominal, sin retornar a las condiciones normales de operación, a pesar de la inyección de potencia reactiva (Indulkar \& Viswanathan, 1983). Denotando que los SPL son mucho más sensibles a variaciones de potencia activa y reactiva, y pueden estar cerca de una inestabilidad de tensión.

Para analizar el efecto de otros parámetros sobre la estabilidad de tensión, en esta publicación serán investigadas la capacidad de cortocircuito del sistema, la reactancia crítica del enlace, el efecto del f.p. de la carga y el ángulo de carga crítico. Finalmente, se evaluará el aporte de la compensación de capacitores en derivación en diferentes puntos de la línea de transmisión.

\section{ANÁLISIS DEL SPL SIN COMPENSAR}

Para analizar el fenómeno del colapso de tensión, se utiliza un SPL, cuyo modelo simplificado se muestra en la Figura 1. Para este modelo, se asume que la carga conectada tiene un f.p. constante.

En este sistema, la línea de transmisión no se encuentra compensada, y la idea es determinar la tensión crítica y el ángulo de potencia crítico que se establecen, cuando el sistema de transmisión alcanza su punto de máxima cargabilidad. 


\subsection{Relaciones de potencia}

Para establecer las relaciones de potencia, se considera el modelo de la Figura 1, a partir del cual, se obtienen las siguientes expresiones de potencia que abastecen la carga.

$P=\frac{E V}{X} \operatorname{sen} \delta$

$Q=\frac{E V}{X} \cos \delta-\frac{V^{2}}{X}$

Al dividir la ecuación (1) entre a la potencia de cortocircuito de la línea, $P c c=E^{2} / X$, se obtienen las ecuaciones adimensionales de las potencias activas y reactivas mostradas en la ecuación (2).

$p=v \cdot \operatorname{sen} \delta$

$q=v \cdot \cos \delta-v^{2}$

donde:

$p=\frac{X}{F^{2}} P$
$q=\frac{X}{F^{2}} Q$

$v=\frac{V}{F}$

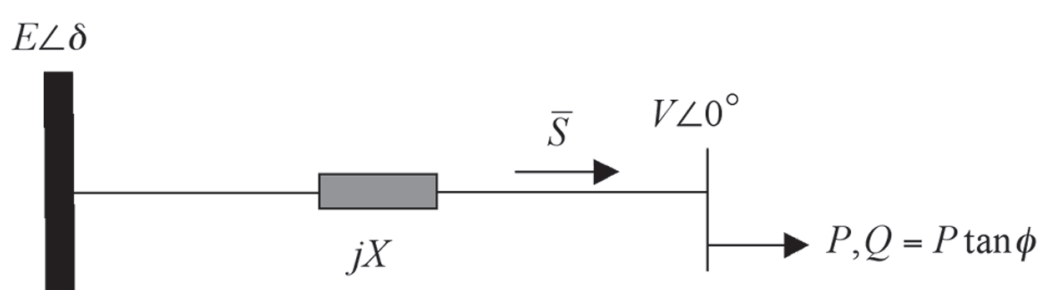

Figura 1. Sistema longitudinal.

Fuente: (el autor).

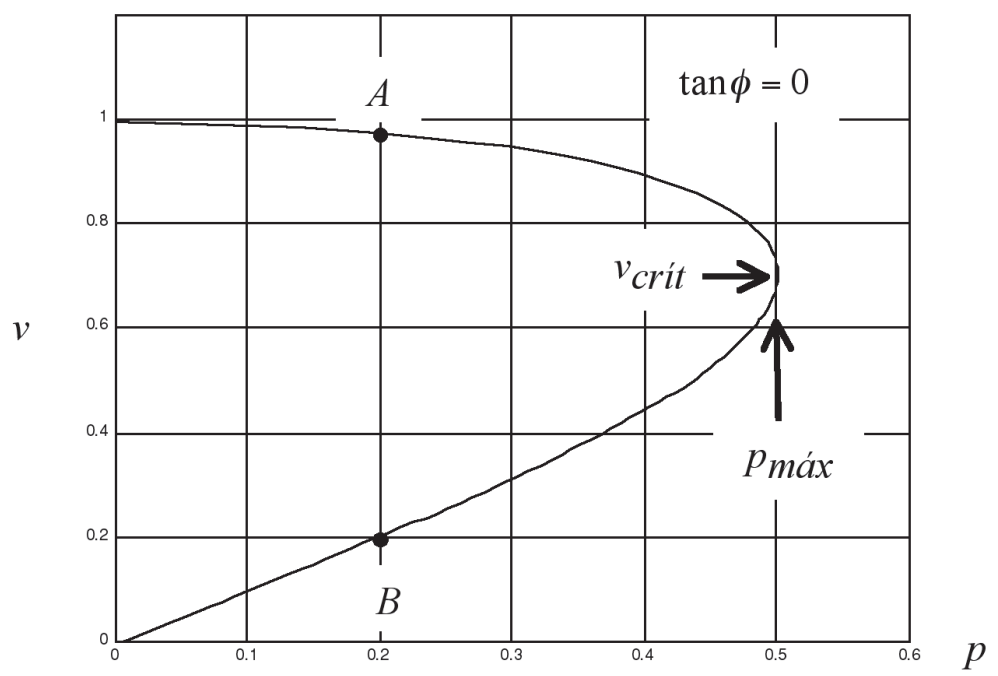

Figura 2. Curva $p$ - v para un f.p. unitario.

Fuente: (el autor). 
En estas ecuaciones, si se elimina la dependencia de $\delta$, se obtiene un polinomio de cuarto orden en $v$, cuya solución con sentido práctico es:

$$
v=\frac{\sqrt{2}}{2} \sqrt{(1-2 q) \pm \sqrt{1-4 p^{2}-4 q}}
$$

En el caso de una carga con f.p. constante, $q=\operatorname{ptan} \phi$, las curvas normalizadas $p-v$ pueden determinarse para diferentes factores de potencia. En el caso de que el f.p. sea unitario, la curva normalizada p - v se muestra en la Figura 2.

A partir de la Figura 2, se verifica el sentido físico de los signos \pm de la ecuación (3). Es decir, hay dos soluciones factibles, en donde el signo positivo corresponde al punto A y el signo negativo corresponde al punto $\mathrm{B}$. Si el sistema se encuentra en el punto de operación A, esto corresponde a una estabilidad de tensión en régimen permanente. Por el contrario, si el sistema se encuentra en el punto de operación B, esto corresponde a una inestabilidad de tensión en régimen permanente.

\subsection{Tensión, potencia y ángulo crítico}

A medida que la demanda de potencia activa se incrementa, el perfil de tensión en la barra de consumo, disminuye, hasta que se alcanza un valor crítico de tensión $v_{\text {crit, }}$ el cual corresponde a la potencia máxima pmáx que puede ser transportada por la línea de transmisión, e indica la condición de máxima cargabilidad del sistema. Este punto de operación vulnerable se reconoce como la nariz de la curva $p$ - $v$ de la Figura 2.

Para determinar el valor de la tensión crítica vcrit, se reconoce que en este punto de operación, la ecuación (3) sólo tiene una única solución, la cual es satisfecha cuando,

$$
\sqrt{1-4 p^{2}-4 q}=0
$$

$\mathrm{Al}$ sustituir las expresiones de la ecuación (2) en (4) y al simplificar los términos, se obtiene una relación entre la tensión crítica $v_{\text {crit }}$ y el ángulo crítico $\delta_{\text {crit }}$, dada por la ecuación (5).

$v_{c r i t}=\frac{1}{2 \cos \delta_{\text {crit }}}$

El mismo resultado anterior puede obtenerse, cuando el flujo de potencia no converge. Analíticamente, esto significa que el determinante de la matriz Jacobiana, ecuación (6), es igual a cero.

$J=\left[\begin{array}{ll}\frac{\partial p}{\partial \delta} & \frac{\partial p}{\partial v} \\ \frac{\partial q}{\partial \delta} & \frac{\partial q}{\partial v}\end{array}\right]=\left[\begin{array}{cc}v \cdot \cos \delta & \operatorname{sen} \delta \\ -v \cdot \operatorname{sen} \delta & (\cos \delta-2 v)\end{array}\right]$

Ahora bien, como la tensión crítica vcrit es una variable independiente en el nodo de carga, el ángulo crítico $\delta_{\text {crit }}$ cambia según se muestra en la Figura 3.

Por otro lado, a partir del sistema de la Figura 1 , se puede deducir una expresión que representa la dependencia entre la potencia reactiva y la tensión, con respecto a la potencia activa, es decir $p=f(v, q)$. Para esto, se elimina la dependencia de $\delta$ mediante (2), dando como resultado:

$p=\sqrt{v^{2}-\left(q+v^{2}\right)^{2}}$

De esta forma, para una carga con un f.p. unitario, la tensión crítica $v_{\text {crit }}$ en ese punto de operación, será un 70,7\% de su valor nominal, y la máxima potencia activa transmitida será un $50 \%$ de su valor nominal. En este caso, el ángulo crítico $\delta_{\text {crit }}$ es igual a $45^{\circ}$.

Por el contrario, si se quiere conocer la dependencia entre la potencia activa y la tensión, con respecto a la potencia reactiva, es decir $q=f(v, p)$, nuevamente, a partir de (2) se obtiene que: 
$q=\sqrt{v^{2}-p^{2}}-v^{2}$

En este caso, cuando la carga tiene un f.p. igual a cero, la tensión crítica vcrit en ese punto de operación, será un 50,0\% de su valor nominal, y la máxima potencia reactiva transmitida será un $25 \%$ de su valor nominal. En este caso, el ángulo crítico dcrit es igual a $0^{\circ}$.

En general, como el f.p. se encuentra entre cero y uno, puede obtenerse una expresión para el ángulo crítico $\delta_{\text {crit }}$, sustituyendo $q$ $=p \tan \phi$ y (5) en (2), y después de efectuar el álgebra correspondiente, se obtiene la ecuación (9).

$\delta_{c r i t}=\frac{\pi}{4}-\frac{\phi}{2}$

A partir de (9) puede evaluarse los siguientes casos extremos:

a. Si el f.p. es unitario, el ángulo crítico es $45^{\circ}$ y la tensión crítica alcanza un valor de 0,707 p.u. b. Si el f.p. es cero, el ángulo crítico es $0^{\circ} \mathrm{y}$ la tensión crítica alcanza un valor de 0,5 p.u.

Para este caso general, la Figura 4 muestra como se comporta el ángulo crítico y el ángulo del f.p., al variar la tensión crítica en el punto de máxima cargabilidad de la línea.

De forma similar, para derivar una expresión genérica de la potencia máxima a transmitir, para cualquier f.p., se sustituyen las ecuaciones (5) y (9) en (2), obteniéndose que,

$p_{\text {máx }}=\frac{1-\tan \frac{\phi}{2}}{2 \cdot\left(1+\tan \frac{\phi}{2}\right)}$

donde el correspondiente valor de potencia reactiva está dado por,

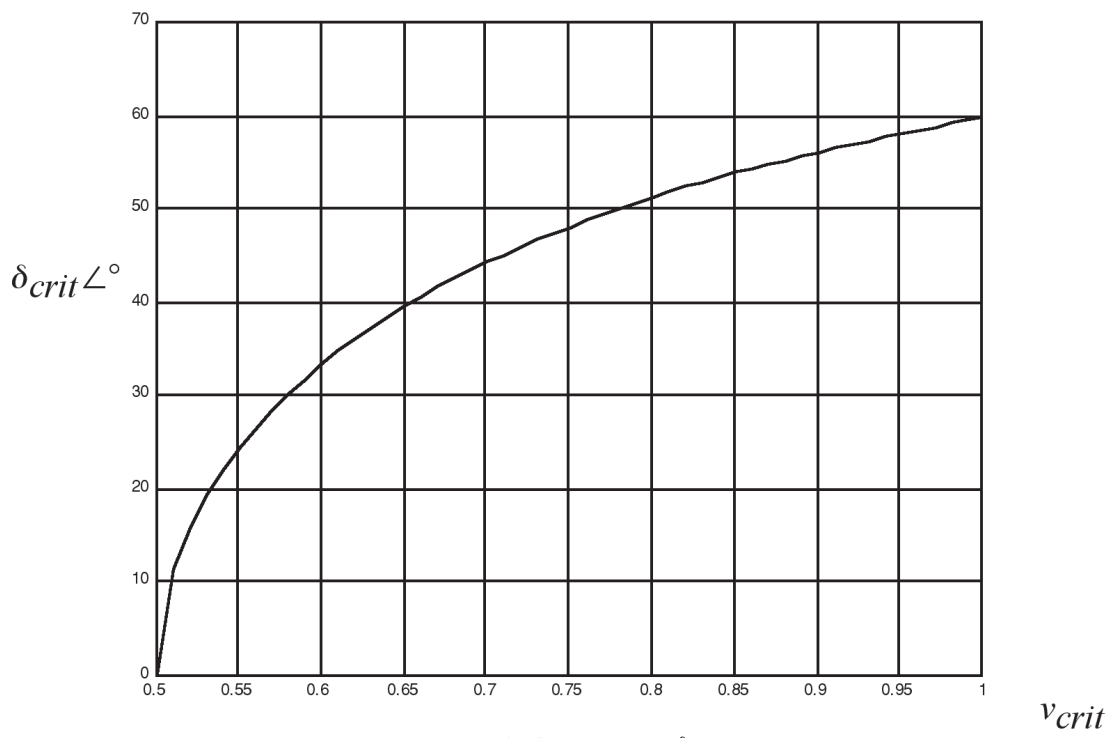

Figura 3. Curva $v_{\text {crit }}-\delta_{\text {crit }}$.

Fuente: (el autor) 


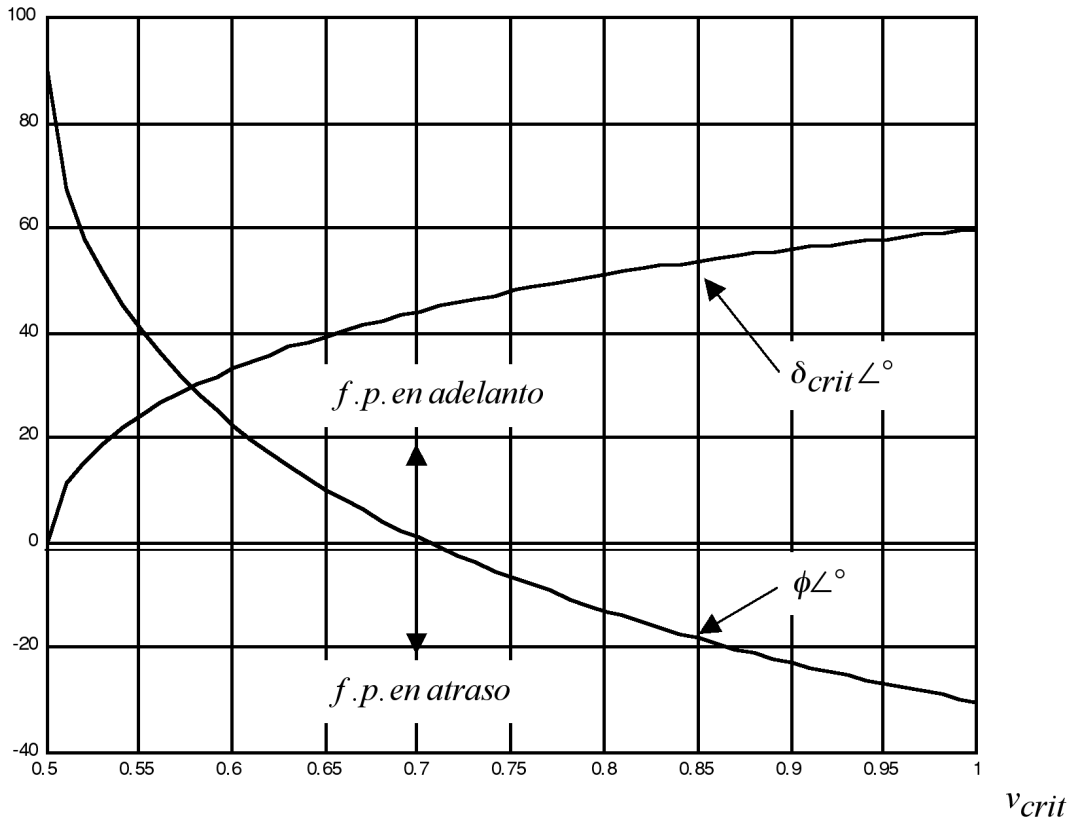

Figura 4. Curvas $v_{\text {crit }}-\delta_{\text {crit }} y v_{\text {crit }}-\phi$.

Fuente: (el autor).

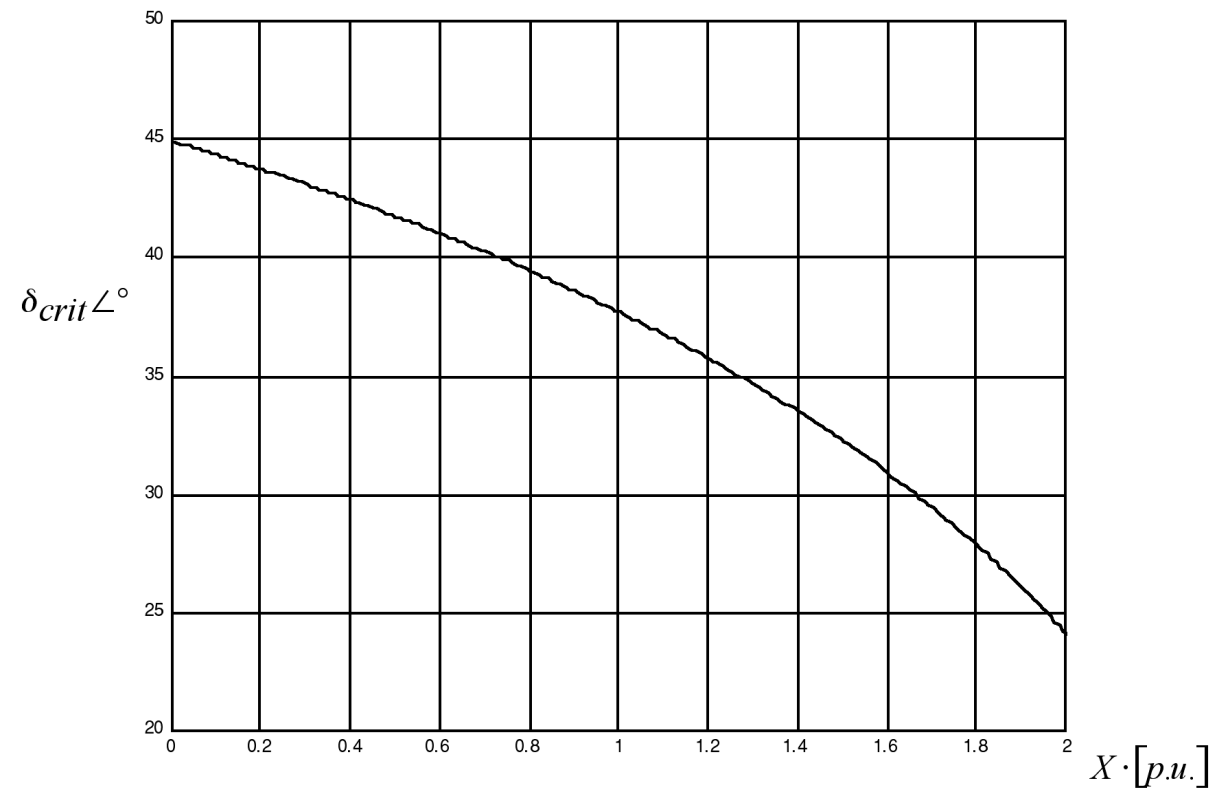

Figura 5. Curva $X-\delta_{\text {crit }}$.

Fuente: (el autor). 


\subsection{Efecto de la reactancia inductiva de la línea}

Para considerar el efecto de la reactancia inductiva de la línea sobre el ángulo crítico, Scrit, y el límite de estabilidad de tensión, Vcrit, se sustituye nuevamente $q=\operatorname{ptan} \phi \mathrm{y}$ (5) en (2), y después de efectuar el álgebra correspondiente, se obtiene la ecuación (12)

$\delta_{\text {crit }}=\cos ^{-1}\left\{\frac{E}{\sqrt{2 E^{2}-4 X P \tan \phi}}\right\}$

Al graficar la ecuación (12), con respecto a la reactancia de la línea y asumiendo que $E=$ 1 p.u. y $Q=0,1$ p.u., se obtiene la Figura 5.

De forma similar, cuando se sustituye la ecuación (12) en la ecuación (5) y el resultado se expresa en unidades naturales, se obtiene la ecuación (13) de la tensión crítica, en función de la reactancia de la línea.

$V_{c r i t}=\frac{\sqrt{2 E^{2}-4 X P \tan \phi}}{2}$

Nuevamente, al graficar la ecuación (13), con respecto a la reactancia de la línea y asumiendo que $E=1$ p.u. y $Q=0,1$ p.u., se obtiene la Figura 6.

\section{ANÁLISIS DEL SPL COMPENSADO}

Para considerar el efecto de la compensación en derivación sobre la tensión, potencia y ángulo crítico en el límite de estabilidad de tensión, se usa el sistema longitudinal de la Figura 7. En este sistema se asume que no hay pérdidas activas y que la carga es de potencia constante.

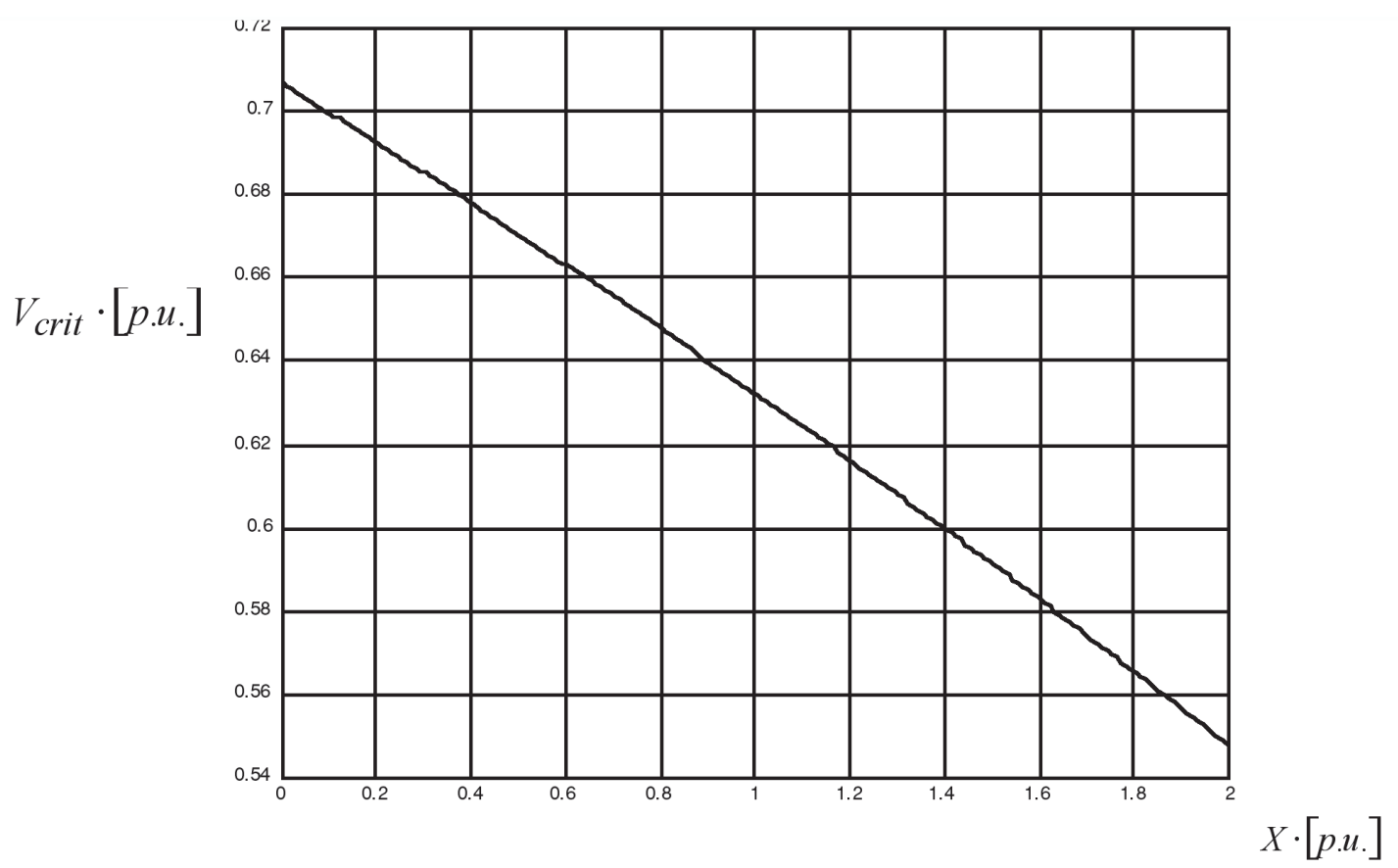

Figura 6. Curva $X-V_{\text {crit }}$.

Fuente: (el autor). 


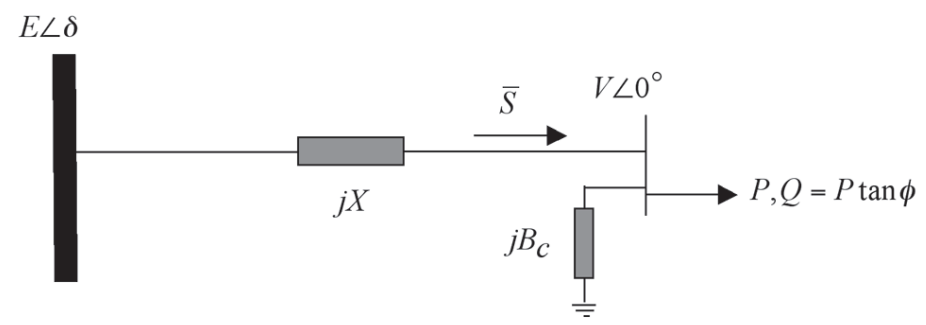

Figura 7. Sistema longitudinal compensado.

Fuente: (el autor).

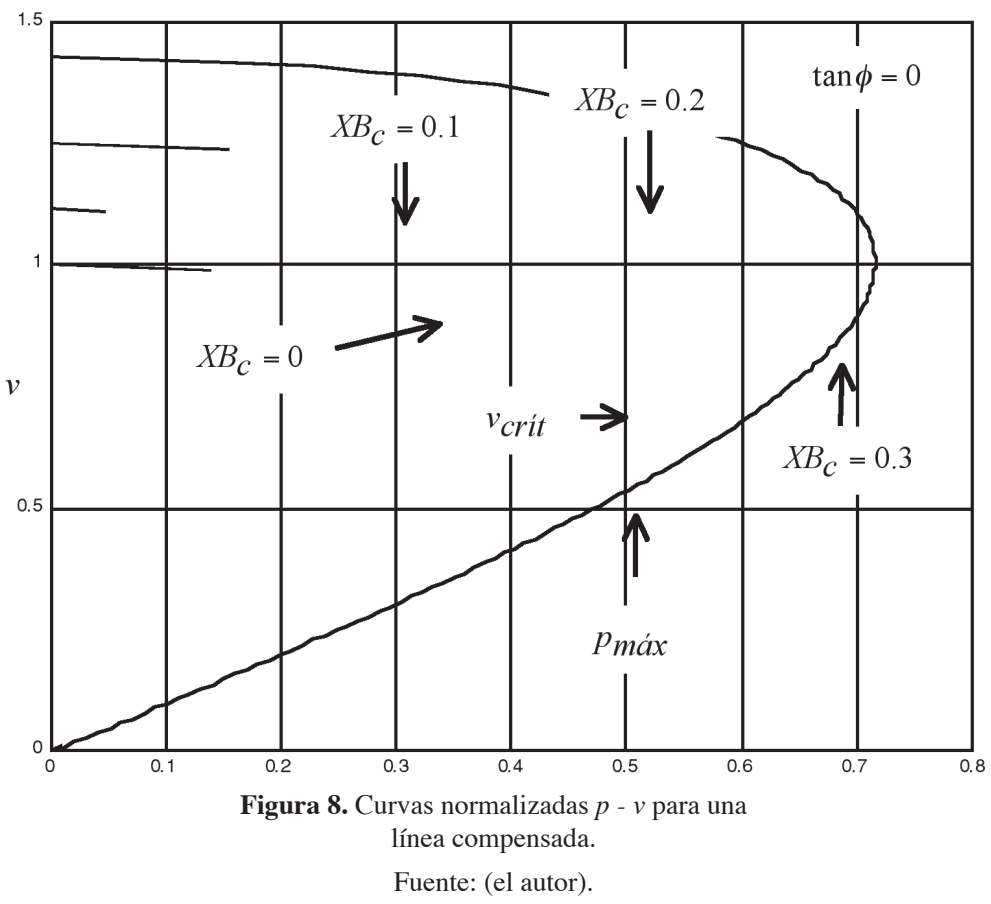

\subsection{Relaciones de potencia}

Las ecuaciones normalizadas del flujo de potencia con respecto a la potencia de cortocircuito, $P_{c c}=E^{2} / X$, se indican en la ecuación (14)

$p=v \cdot \operatorname{sen} \delta$

$q=v \cdot \cos \delta-\left(1-X B_{c}\right) \cdot v^{2}$

De nuevo, similar al caso no compensado, al eliminar la dependencia de $\delta$ en (14), se obtiene un polinomio de cuarto orden en $v$, cuya solución con sentido práctico es:
Para visualizar como la compensación en derivación modifica las condiciones de las curvas normalizadas $p-v$, se asume una carga de f.p. unitario. En este caso, las curvas normalizadas $p-v$ para diferentes grados de compensación, se muestran en la Figura 8.

De la Figura 8 , se observa como la compensación incrementa la potencia máxima transmitida, así como aumenta el valor de la tensión crítica, desplazando la nariz de la curva $p-v$ a la derecha y hacia arriba. Con este suministro de potencia reactiva en el extremo receptor de la línea, se incrementa el margen de potencia reactiva de la barra, y se evita un colapso de tensión ante alguna contingencia en el sistema. 
$v=\frac{\sqrt{2}}{2 \cdot\left(1-X B_{c}\right)} \sqrt{\left(1-2 q\left(1-X B_{c}\right)\right) \pm \sqrt{1-4 p^{2}\left(1-X B_{c}\right)^{2}-4 q\left(1-X B_{c}\right)}}$

\subsection{Tensión, potencia y ángulo crítico}

Para determinar el valor de la tensión crítica vcrit, se reconoce que en este punto de operación, la ecuación (15) sólo tiene una única solución, la cual es satisfecha cuando,

$\sqrt{1-4 p^{2}\left(1-X B_{c}\right)^{2}}=0$

Al sustituir las expresiones de la ecuación (14) en (16) y al simplificar los términos, se obtiene una relación entre la tensión crítica vcrit, y el ángulo crítico ঠcrit, dada por la ecuación (17).

$v_{c r i t}=\frac{1}{2 \cdot\left(1-X B_{c}\right) \cdot \cos \delta_{c r i t}}$

La potencia máxima transmitida cuando el sistema alcanza el valor de la tensión crítica, se evalúa cuando el determinante de la matriz
Jacobiana de las ecuaciones de flujo de potencia, es igual a cero. En otras palabras, cuando el flujo de potencia no converge, la condición de máxima cargabilidad, correspondiente a una carga con f.p. unitario, está dada por la ecuación (18).

$p_{\text {máx }}=\frac{1}{2 \cdot\left(1-X B_{c}\right)}$

Por otro lado, la ecuación (17) permite obtener la familia de curvas de la Figura 9, según sea el grado de compensación.

Para el caso de la línea compensada, el ángulo crítico Scrit, es independiente del grado de compensación y es igual al esquema de la línea no compensada, ecuación (9).

De forma similar, para derivar una expresión genérica de la potencia máxima a transmitir, para cualquier f.p., se sustituyen las ecuaciones (17) y (9) en (14), obteniéndose que,

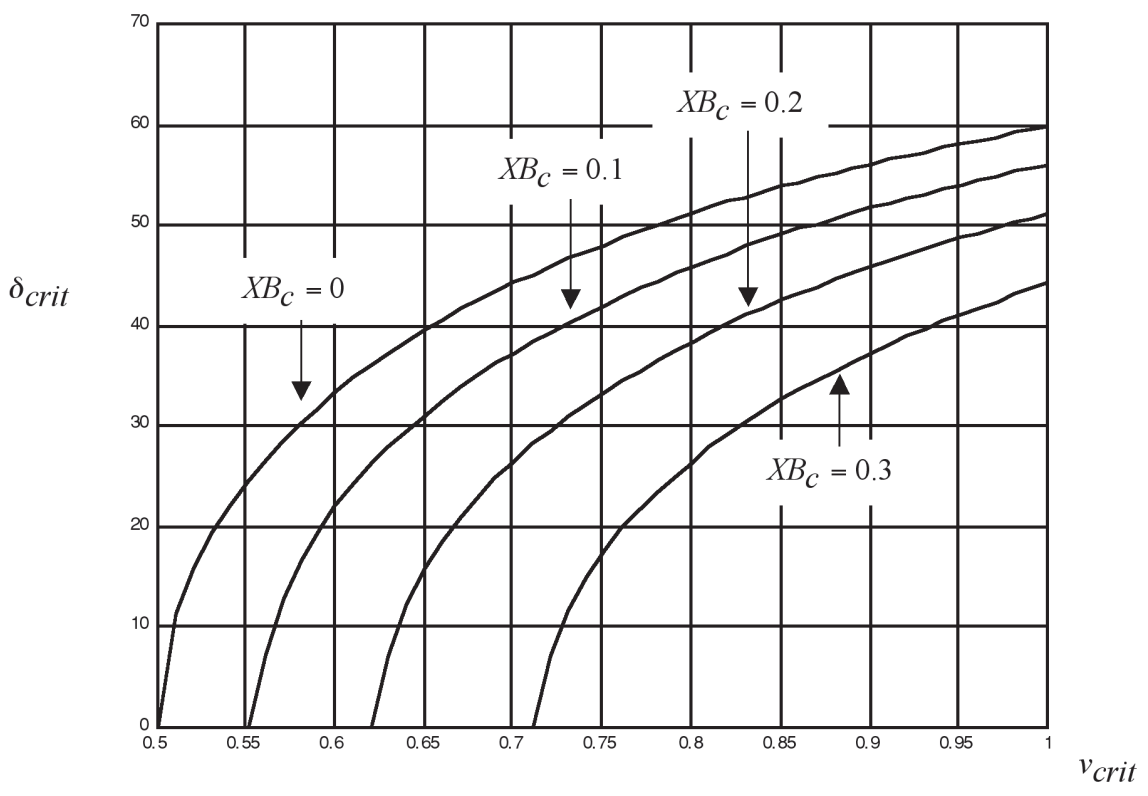

Figura 9. Curvas $v_{\text {crit }}-\delta_{\text {crit }}$ para la línea compensada.

Fuente: (el autor). 


$$
p_{\text {máx }}=\frac{1-\tan \frac{\phi}{2}}{2 \cdot\left(1-X B_{c}\right)\left(1+\tan \frac{\phi}{2}\right)}
$$

donde el correspondiente valor de potencia reactiva está dado por,

$q=\frac{0.25-p_{\operatorname{máx}}}{1-X B_{c}}$

\subsection{Efecto de la reactancia inductiva}

\section{de la línea}

Para considerar el efecto de la reactancia inductiva de la línea sobre el ángulo crítico, ¿crit, y el límite de estabilidad de tensión, Vcrit, se sustituye nuevamente $\mathrm{q}=\operatorname{ptan} \phi$ y (17) en (14), y después de efectuar el álgebra correspondiente, se obtiene la ecuación (21):

$\delta_{c r i t}=\cos ^{-1}\left\{\frac{E}{\sqrt{2 E^{2}-4 X\left(1-X B_{c}\right) P \tan \phi}}\right\}$
De forma similar, cuando se sustituye la ecuación (21) en la ecuación (17) y el resultado se expresa en unidades naturales, se obtiene la ecuación (22) de la tensión crítica, en función de la reactancia de la línea.

$$
V_{c r i t}=\frac{\sqrt{2 E^{2}-4 X\left(1-X B_{c}\right) P \tan \phi}}{2 \cdot\left(1-X B_{c}\right)}
$$

\section{ANÁLISIS DE LA ESTABILIDAD DE TENSIÓN EN UNA LÍNEA COMPENSADA EN CUALQUIER SITIO}

Si la compensación de potencia reactiva se efectúa en cualquier sitio de la línea de enlace, el modelo a obtener se muestra en la Figura 10.

\subsection{Relaciones de potencia}

Las ecuaciones normalizadas del flujo de potencia con respecto a la potencia de cortocircuito, Pcc=E2/X, se indican en la ecuación (23).

$p=v \cdot \operatorname{sen} \delta$

$q=v \cdot \cos \delta-\left(1-X_{1} B_{c}\right) \cdot v^{2}+X_{2} B_{c}$

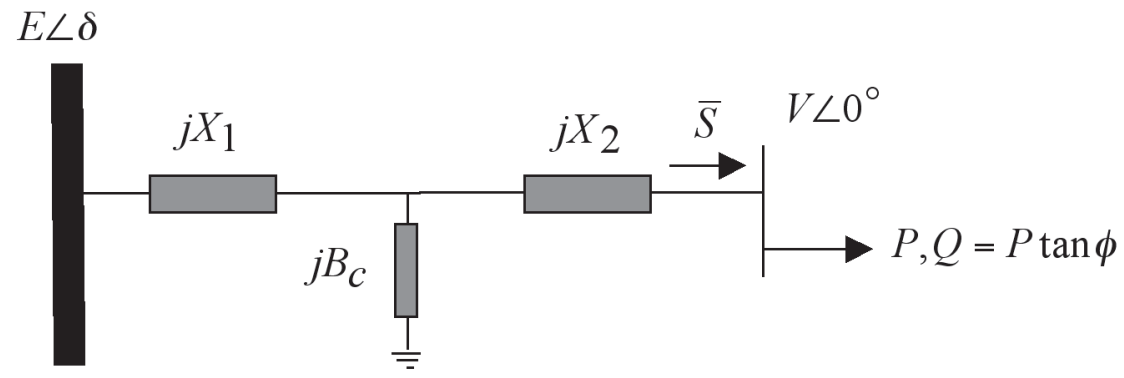

Figura 10. Sistema longitudinal compensado.

Fuente: (el autor) 


\subsection{Tensión, potencia y ángulo crítico}

Para determinar el valor de la tensión crítica vcrit, se evalúa el punto de operación, en el cual el determinante de la matriz Jacobiana, ecuación (24), es igual a cero.

$J=\left[\begin{array}{cc}v \cdot \cos \delta & \operatorname{sen} \delta \\ -v \cdot \operatorname{sen} \delta & \left(\cos \delta-2\left(1-X_{1} B_{c}\right) \cdot v\right)\end{array}\right]$

En este punto de operación se obtiene que,

$v_{c r i t}=\frac{1}{2 \cdot\left(1-X_{1} B_{c}\right) \cdot \cos \delta_{c r i t}}$

De forma similar, para derivar una expresión genérica de la potencia máxima a transmitir, para cualquier $\delta_{\text {crit }}$, se sustituyen las ecuaciones (25) en (23), obteniéndose que,

$p_{\text {máx }}=\frac{\tan \delta_{\text {crit }}}{2 \cdot\left(1-\left(X_{1}+X_{2}\right) B_{c}\right)}$

donde el correspondiente valor de potencia reactiva está dado por,

$q=\frac{0.25-p_{\text {máx }}}{1-\left(X_{1}+X_{2}\right) B_{c}}+X_{2} B_{c}$

\subsection{Efecto de la reactancia inductiva de la línea}

Para considerar el efecto de la reactancia inductiva de la línea sobre el ángulo crítico, ঠcrit, y el límite de estabilidad de tensión, Vcrit, se sustituye nuevamente $q=\operatorname{ptan} \phi$ y (25) en (23), obteniéndose la ecuación (28)

De forma similar, cuando se sustituye la ecuación (28) en la ecuación (25) y el resultado se expresa en unidades naturales, se obtiene la ecuación (29) de la tensión crítica, en función de la reactancia de la línea.

donde

$X_{12}=X_{1}+X_{2}-X_{1} X_{2} B_{1}$

\section{CONCLUSIONES}

De los resultados obtenidos, se desprenden las siguientes conclusiones:

1. La máxima transferencia de potencia activa se encuentra limitada por el f.p. de la carga, la reactancia de la línea y el desfase entre las tensiones nodales de los extremos de la línea.

2. El desfase crítico entre las tensiones nodales de los extremos de la línea, es independiente del grado de compensación, si ésta se efectúa en el extremo receptor de la línea.

3. La localización de la compensación estática de potencia reactiva, afecta el margen de estabilidad de tensión.

$\delta_{c r i t}=\cos ^{-1}\left\{\frac{E}{\sqrt{2 E^{2}+4 X\left(1-X_{1} B_{c}\right)\left(X_{2} B_{c} E^{2}-X_{12} P \tan \phi\right)}}\right\}$

$v_{\text {crit }}=\frac{\sqrt{2 E^{2}+4 X\left(1-X_{1} B_{c}\right)\left(X_{2} B_{c} E^{2}-X_{12} P \tan \phi\right)}}{2 \cdot\left(1-X_{1} B_{c}\right)}$ 


\section{REFERENCIAS BIBLIOGRÁFICAS}

Obadina, O. O. \& Berg, G. J. (1990, May.). Identifying Electrically Weak and Strong Segments of a Power System from a Voltage Stability Viewpoint. IEE Proceedings, 137(3), 205-212.

Abdel, A. (1987, August). Controllable VAR Compensation: a Potential Solution to Loadability Problems of Low Capacity Power Systems. IEEE Trans. Power Systems, 2(3), 561-567.

Aboytes, F. \& Arroyo, G. (1986, May.). Security Assessment in the Operation of Longitudinal Power Systems. IEEE Trans. Power Systems, 1(2), 225-231.

Instituto Costarricense de Electricidad (ICE), Centro Nacional de Control de Energía. (2001, Marzo.). Informe del Apagón Nacional del 6 de marzo del Año 2001. San José, Costa Rica.

Indulkar, C. S. \& Viswanathan, B. (1983, July.). Deterministic and Probabilistic Approach to Voltage Stability of Series Compensators EHV Transmission Lines. IEEE Trans. PowerApparatus and Systems, 102(7), 2317-2323.

Stubbe, M., Bihain, A. \& Deuse, J. (1993, May). Simulation of Voltage Collapse.
Electrical Power \& Energy Systems, 15(4), 239-243.

Taylor, C. W. (1994). Power Systems Voltage Stability. New York: McGraw-Hill, 66-81.

\section{SIMBOLOGÍA}

E: $\quad$ Tensión en el extremo emisor de la línea

$V: \quad$ Tensión en el extremo receptor de la línea

$X: \quad$ Reactancia inductiva de la línea

P: $\quad$ Potencia activa demandada por la carga

$Q: \quad$ Potencia reactiva demandada por la carga.

$\delta$ : $\quad$ Ángulo de potencia del sistema

$\cos \phi: \quad$ Factor de potencia de la carga.

\section{SOBRE EL AUTOR}

\section{Eddie A. Araya Padilla}

Catedrático de la Escuela de Ingeniería Eléctrica, Universidad de Costa Rica.

Sus áreas de interés son la dinámica no lineal, los dispositivos FACTS y la generación eólica.

Correo electrónico: earaya@eie.ucr.ac.cr 